Proyecciones Journal of Mathematics

Vol. 33, $\mathrm{N}^{o}$ 1, pp. 25-41, March 2014.

Universidad Católica del Norte

Antofagasta - Chile

\title{
Global neighbourhood domination
}

\author{
S. V. Siva Rama Raju \\ Ibra College of Technology, Sultanate of Oman \\ and \\ I. H. Nagaraja Rao \\ G. V. P. P. G. Courses, India \\ Received : September 2012. Accepted : October 2013
}

\begin{abstract}
A subset $D$ of vertices of a graph $G$ is called a global neighbourhood dominating set(gnd - set) if $D$ is a dominating set for both $G$ and $G^{N}$, where $G^{N}$ is the neighbourhood graph of $G$. The global neighbourhood domination number(gnd - number) is the minimum cardinality of a global neighbourhood dominating set of $G$ and is denoted by $\gamma_{g n}(G)$. In this paper sharp bounds for $\gamma_{g n}$, are supplied for graphs whose girth is greater than three. Exact values of this number for paths and cycles are presented as well. The characterization result for a subset of the vertex set of $G$ to be a global neighbourhood dominating set for $G$ is given and also characterized the graphs of order $n$ having gnd numbers $1,2, n-1, n-2, n$.
\end{abstract}

Subject Classification : 05C69.

Keywords : Global neighbourhood domination, global neighbourhood domination number, global domination, restrained domination, connected domination. 


\section{Introduction \& Preliminaries}

Domination is an active subject in graph theory, and has numerous applications to distributed computing, the web graph and adhoc networks. For a comprehensive introduction to theoretical and applied facets of domination in graphs the reader is directed to the book [4].

A set $D$ of vertices is called a dominating set of $G$ if each vertex not in $D$ is joined to some vertex in $D$. The domination number $\gamma(G)$ is the minimum cardinality of the dominating set of $G[4]$.

Many variants of the domination number have been studied. For instance a dominating set $S$ of a graph $G$ is called a restrained dominating set if every vertex in $V-D$ is adjacent to a vertex in $D$ as well as another vertex in $V-D$. The restrained domination number of $G$, denoted by $\gamma_{r}(G)$ is the smallest cardinality of the restrained dominating set of $G$ [3]. A set $D$ is called a global dominating set of $G$ if $D$ is a dominating set of both $G$ and its complement $G^{c}$. The global domination number of $G$, denoted by $\gamma_{g}(G)$ is the smallest cardinality of the global dominating set of $G$ [6]. A dominating set $D$ of connected graph $G$ is called a connected dominating setof $G$ if the induced subgraph $\langle D\rangle$ is connected. The connected domination number of $G$, denoted by $\gamma_{c}(G)$ is the smallest cardinality of the connected dominating set of $G$ [7]. A dominating set $D$ of connected graph $G$ is called an independent dominating set of $G$ if the induced subgraph $<D>$ is a null graph [4].

$G$ be a connected graph, then the Neighbourhood Graph of $G$ is denoted by $N(G)$ (or) $G^{N}$ and it has the same vertex set as that of $G$ and edge set being $\{u v / u, v \in V(G)$, there is $w \in V(G)$ such that $u w, w v \in E(G)\}$ [2].

Recently we have introduced a new type of graph known as semi complete graph. Let $G$ be a connected graph, then $G$ is said to be semi complete if any pair of vertices in $G$ have a common neighbour. The necessary and sufficient condition for a connected graph to be semi complete is any pair of vertices lie on the same triangle or lie on two different triangles having a common vertex [5].

In the present paper, we introduce a new graph parameter, the global neighbourhood domination number, for a connected graph $G$. We call $D \subseteq V(G)$ a global neighbourhood dominating set (gnd - set) of $G$ if $D$ is a dominating set for both $G, G^{N}$. The global neighbourhood domination number is the minimum cardinality of a global neighbourhood dominating set of $G$ and is denoted by $\gamma_{g n}(G)$. 
Example. Suppose $G$ is a graph representing a network of roads linking various locations. Some essential goods are being supplied to these locations from supplying stations. It may happen that these links(edges of $G)$ may be broken for some reason or the other. So we have to think of maintaining the supply of goods to various locations uninterrupted through secret links(edges in the neighbourhood graph of $G$ ). As the neighbourhood graph of $G$ is a spanning subgraph of $G^{c}$, the construction (maintainance) cost of secret links can be minimized, when compared with the complementary graph of $G$. The global neighbourhood domination number will be the minimum number of supplying stations needed to accomplish the task of supplying the goods uninterruptedly.

All graphs considered in this paper are simple, finite, undirected and connected. For all graph theoretic terminology not defined here, the reader is referred to [1].

In section [2], sharp bounds for $\gamma_{g n}$ are supplied for the graphs whose girth is greater than three. In section [3], we have given a characterization result for a proper subset of the vertex set of $G$ to be a gnd - set of $G$ and also characterized the graphs whose gnd - numbers are 1,2,n, n-1, n-2.

\section{Bounds for the global neighbourhood domination number}

In this section, we obtain some bounds for the gnd - numbers of graphs whose girth is greater than three.

Theorem 2.1. If $G$ is a triangle free graph, then

$$
\frac{2 e-n(n-3)}{2} \leq \gamma_{g n}(G) \leq n-\Delta(G)+1
$$

Proof: Let $D$ be a minimum gnd - set of $G$. By hypothesis every vertex in $V-D$ is non adjacent with atleast one vertex in $D$. Otherwise we get a contradiction to that $D$ is a gnd - set for $G$.

$$
\begin{aligned}
& \Rightarrow e \leq \frac{n(n-1)}{2}-\left[n-\gamma_{g n}(G)\right] \\
& \Rightarrow \frac{2 e-n(n-3)}{2} \leq \gamma_{g n}(G) \quad \rightarrow(1)
\end{aligned}
$$

Suppose that $d_{G}(v)=\Delta(G)$ for some $v$ in $V(G)$. 
Let $v_{1}, v_{2}, \ldots, v_{\Delta(G)}$ be the neighbours of $v$ in $G$. Then

$\left[V-\left\{v_{1}, v_{2}, \ldots, v_{\Delta(G)}\right\}\right] \bigcup\left\{v_{i}: i\right.$ is one of $\left.1,2, \ldots, \Delta(G)\right\}$ is a gnd - set of $G$ and its cardinality is $n-\Delta(G)+1$.

$$
\Rightarrow \gamma_{g n}(G) \leq n-\Delta(G)+1 \quad \rightarrow(2)
$$

From (1) and (2)

$$
\frac{2 e-n(n-3)}{2} \leq \gamma_{g n}(G) \leq n-\Delta(G)+1 .
$$

Furthermore the lower bound is attained in the case of $C_{4}$ and upper bound is attained in the case of $P_{3}$. Hence the bounds are sharp.

Note: The upper bound holds good for any connected graph $G$.

1. $\gamma_{g n}\left(K_{n}\right)=1 ; n \geq 3$

2. $\gamma_{g n}\left(S_{n}\right)=2 ; n \geq 3$

3. $\gamma_{g n}\left(K_{m, n}\right)=2 ; m+n \geq 3$

4. $\gamma_{g n}\left(P_{n}\right)=\left[\frac{n}{3}\right] \quad ; n=3 m+1$

$$
=\left[\frac{n}{3}\right]+2 ; n=3 m, 3 m+2
$$

Here $n \geq 4$.

5. $\gamma_{g n}\left(C_{n}\right)=\left[\frac{n}{3}\right] \quad ; n=3 m$

$$
=\left[\frac{n}{3}\right]+1 ; \quad n=3 m+1,3 m+2
$$

6. $\gamma_{g n}\left(C_{n} O K_{2}\right)=n$.

$G=P_{n}(n \geq 4)$. Then there is an independent gnd - set for $G$ iff $n=3 m+1$.

$G=C_{n}(n \geq 3)$. Then there is an independent gnd - set for $G$ iff $n=3 m$.

$G=P_{n}(n \geq 3)$. Then $\gamma_{g n}(G)=n-2$ iff $n=4,5$.

$G=C_{n}(n \geq 3)$. Then $\gamma_{g n}(G)=n-2$ iff $n=3,4,5$.

If $T$ is a tree of order $n \geq 3$, then $\gamma_{g n}(G)=2$ iff $T$ is obtained from $P_{3}$ or $P_{4}$ by adding zero or more leaves to the stems of the path. 
Theorem 2.2. $G$ be a connected graph and $D$ be a minimum dominating set of $G$. If there is a vertex $v$ in $V-D$ such that $v$ is adjacent to all the vertices in $D$, then $\gamma_{g n}(G) \leq 1+\gamma(G)$.

Proof: Assume that $D \subseteq N_{G}(v)$ for some $v \in V$. The proof follows from the fact that $D \bigcup\{v\}$ is a gnd - set of $G$.

Theorem 2.3. $D$ be a minimum dominating set of $G$. Then $\gamma_{g n}(G)=$ $1+\gamma(G)$ iff there is a vertex $v$ in $V-D$ satisfying:

(i) $N(v) \subset D$, each of the vertices in $N(v)$ is isolated in $\langle D\rangle$.

(ii) $v_{1} \in V-D\left(v \neq v_{1}\right)$ satisfies (i) then $N(v) \bigcap N\left(v_{1}\right) \neq \phi$.

Proof: Assume that $\gamma_{g n}(G)=1+\gamma(G)$. Then there is a vertex $v$ in $V-D$ satisfying (i) and (ii), otherwise $\gamma_{g n}(G)=\gamma(G)$ which is a contradiction.

Assume that the converse holds. Then $D \bigcup\{v\}$ is a gnd - set in $G$ and $D$ is not a gnd - set in $G$. Thus $D \bigcup\{v\}$ is a minimum gnd - set in $G$.

Hence $\gamma_{g n}(G)=|D \bigcup\{v\}|=\gamma(G)+1$.

Theorem 2.4. $G$ be a connected graph, then $\gamma(G) \leq \gamma_{g n}(G) \leq \gamma_{c}(G)$.

Proof: Clearly $\gamma(G) \leq \gamma_{g n}(G)$. Since any connected dominating set for $G$ is a gnd - set for $G, \gamma_{g n}(G) \leq \gamma_{c}(G)$. Hence $\gamma(G) \leq \gamma_{g n}(G) \leq \gamma_{c}(G)$.

Theorem 2.5. $G$ be a connected graph with $g(G)>3$, then $\gamma_{g}(G) \leq$ $\gamma_{g n}(G)$.

Proof: By hypothesis, every gnd - set is a global dominating set in $G$. Hence $\gamma_{g}(G) \leq \gamma_{g n}(G)$.

Note: Under the hypothesis given in the Theorem(2.10) and Theorem(2.9), we have

$$
\gamma(G) \leq \gamma_{g}(G) \leq \gamma_{g n}(G) \leq \gamma_{c}(G)
$$


Theorem 2.6. $G$ be a connected graph. Then $G=G^{N N}$ iff

(i) Each edge in $G$ lies on $C_{3}$ or $C_{5}$.

(ii) There is no path of length four between any pair of non adjacent vertices in $G$.

Proof: Assume that $G=G^{N N}$.

(i) Let $v_{1}, v_{2}$ be an arbitrary edge in $G$, then by our assumption $v_{1} v_{2}$ is an edge in $G^{N N}$.

Suppose $v_{1} v_{2} \in G^{N}$. Since $v_{1} v_{2} \in E(G), v_{1} v_{2}$ lies on a cycle $C_{3}$ in $G$.

Suppose $v_{1} v_{2} \notin G^{N}$. Since $v_{1} v_{2} \in G^{N N}$, there is a $v_{3}$ in $V(G)$ such that $<v_{1} v_{3} v_{2}>$ is a path in $G^{N}$. This implies $v_{1} v_{3}, v_{3} v_{2} \in E\left(G^{N}\right)$. So there is a path of length four from $v_{1}$ to $v_{2}$ in $G$. Thus $P \bigcup\left\{v_{1} v_{2}\right\}$ is a 5 - cycle in $G$. Therefore $v_{1} v_{2}$ lies on $C_{5}$.

Hence each edge in $G$ lies on $C_{3}$ or $C_{5}$.

(ii) If there is a path of length four between any pair of non adjacent vertices in $G$, then there is an edge in $G^{N N}$ which is not in $G$. Hence $G \neq G^{N N}$, which is a contradiction.

Assume that the converse holds.

Let $v_{1} v_{2}$ be an arbitrary edge in $G$. Then by (i) of our assumption $v_{1} v_{2}$ lies on $C_{3}$ or $C_{5}$. In either case $v_{1} v_{2}$ is an edge in $G^{N N}$. Hence $G \subseteq G^{N N}$.

Let $v_{1} v_{2} \in E\left(G^{N N}\right)$. Suppose that $v_{1} v_{2} \notin E(G)$. In either case $v_{1} v_{2} \in$ $E\left(G^{N}\right)$ or $v_{1} v_{2} \notin E\left(G^{N}\right)$ there is a path of length four from $v_{1}$ to $v_{2}$ in $G$, which is a contradiction. Thus $v_{1} v_{2} \in E(G)$. Thus $G^{N N} \subseteq G$.

Hence $G^{N N}=G$.

If $G$ is a graph satisfying conditions (i) and (ii) of Theorem(2.12), then

$$
\frac{\gamma+\bar{\gamma}}{2} \leq \gamma_{g n} \leq \gamma+\bar{\gamma}
$$

Proof: Under the given hypothesis by Theorem $(2.12), \gamma_{g n}=\bar{\gamma}_{g n}$.

We have $\gamma \leq \gamma_{g n}, \bar{\gamma} \leq \bar{\gamma}_{g n}=\gamma_{g n}$ 


$$
\begin{aligned}
& \Rightarrow \quad \gamma+\bar{\gamma} \leq 2 \gamma_{g n} \\
& \Rightarrow \quad \frac{\gamma+\bar{\gamma}}{2} \leq \gamma_{g n} \rightarrow(1)
\end{aligned}
$$

Clearly,

$$
\gamma_{g n} \leq \gamma+\bar{\gamma} \quad \rightarrow(2)
$$

From (1) and (2),

$$
\frac{\gamma+\bar{\gamma}}{2} \leq \gamma_{g n} \leq \gamma+\bar{\gamma}
$$

Here $\gamma=\gamma(G), \gamma_{g n}=\gamma_{g n}(G), \bar{\gamma}=\gamma_{g n}\left(G^{N}\right)$.

\section{Characterization and Other Relevant Results.}

In this section we have given the characterization for a proper subset of the vertex set of a graph to be a gnd - set.

Theorem 3.1. (Characterization Result) $G$ be a connected graph. $D \subset V$ is a gnd - set of $G$ iff each vertex in $V-D$ lies on an edge whose end points are totally dominated by the vertices in $D$.

Proof: Assume that $D$ is a gnd - set for $G$. Let $v_{1} \in V-D$.

Since $D$ is a dominating set for $G$ there is a $v_{2} \in D$ such that $v_{1} v_{2} \in$ $E(G)$.

Since $D$ is a dominating set for $G^{N}$ there is a $v_{3} \in D$ such that $<v_{1} v_{4} v_{3}>$ is a path in $G$ for some $v_{4} \in V(G)$.

If $v_{4} \neq v_{2}$, then $v_{1}$ lies on the edge $v_{1} v_{4}$, where $v_{1}$ is dominated by $v_{2}$ and $v_{4}$ is dominated by $v_{3}\left(v_{2}, v_{3} \in D\right)$.

If $v_{4}=v_{2}$, then $v_{1}$ lies on the edge $v_{1} v_{2}$, where $v_{1}$ is dominated by $v_{2}$ and $v_{2}$ is dominated by $v_{3}\left(v_{2}, v_{3} \in D\right)$.

So in either case $v_{1}$ lies on the edge whose end points are totally dominated by the vertices in $D$.

Assume that the converse holds. 
Let $v_{1} \in V-D$. Then by our assumption there is a $v_{2} \in V(G), v_{3}, v_{4} \in D$ such that $v_{1} v_{3}, v_{2} v_{4} \in E(G)$.

case:(i) Suppose $v_{2}=v_{3}$.

Then $<v_{1} v_{2} v_{4}>$ is a path in $G$

$\Rightarrow v_{1} v_{4} \in E\left(G^{N}\right), v_{4} \in D$

case:(ii) Suppose $v_{2} \neq v_{3}$.

Then $<v_{3} v_{1} v_{2} v_{4}>$ is a path in $G$

$\Rightarrow v_{1} v_{4} \in E\left(G^{N}\right), v_{4} \in D$.

Therefore $v_{1}$ is dominated by $v_{3}$ in $G$ and by $v_{4}$ in $G^{N}$.

Since $v_{1}$ is arbitrary, $D$ is a gnd - set of $G$.

Theorem 3.2. $G$ be a connected graph. Then $G^{N}$ is complete iff $G$ is semi-complete.

Proof: Assume that $G^{N}$ is complete.

Let $v_{1}, v_{2} \in V(G)$. Since $G^{N}$ is complete, $v_{1} v_{2} \in E\left(G^{N}\right)$. Then there is a $v_{3}$ in $V(G)-\left\{v_{1}, v_{2}\right\}$ such that $\left\langle v_{1} v_{3} v_{2}>\right.$ is a path in $G$.

Hence $G$ is semi complete.

Assume that the converse holds.

Let $v, v_{2} \in V(G)$. By our assumption, there is a $v_{3}$ in $G$ such that $<v_{1} v_{3} v_{2}>$ is a path in $G$

$\Rightarrow v_{1} v_{2} \in E\left(G^{N}\right)$.

Hence $G^{N}$ is complete. $G$ be a connected graph. Then $\gamma_{g n}(G)=1$ iff $G$ is semi-complete and $\gamma(G)=1$.

Proof: Assume that $\gamma_{g n}(G)=1$. 
$\left.\Rightarrow \gamma_{(} G\right)=1, \gamma_{g n}(G)=1$.

Then there is a $v_{0} \in V$ such that $D=\left\{v_{0}\right\}$ is a dominating set for $G, G^{N}$.

Now we show that $G$ is semi complete.

Let $v_{1}, v_{2} \in V(G)$.

case:(i) Suppose $v_{1} \neq v_{0} \neq v_{2}$.

subcase:(a) Suppose $v_{1} v_{2} \in E(G)$.

Clearly $<v_{1} v_{0} v_{2}>$ is a path in $G$. Thus $<v_{1} v_{0} v_{2}>\bigcup\left\{v_{1} v_{2}\right\}$ is a triangle in $G$.

Hence $v_{1} v_{2}$ lie on the same triangle in $G$.

subcase:(b) Suppose $v_{1} v_{2} \notin E(G)$.

Clearly $<v_{1} v_{0} v_{2}>$ is a path in $G$. Since $v_{1} v_{0} \in E(G)$ and $\left\{v_{0}\right\}$ is a dominating set of $G^{N}$ there is a $v_{3} \in V-\left\{v_{0}, v_{1}\right\}$ such that $<v_{1} v_{3} v_{0} v_{1}>$ is a triangle in $G$.

Similarly for $v_{0} v_{2}$ in $E(G)$, there is $v_{4}$ in $V-\left\{v_{0}, v_{2}\right\}$ such that $<$ $v_{2} v_{0} v_{4} v_{2}>$ is a triangle in $G$.

Hence $v_{1}, v_{2}$ lie on triangles $<v_{1} v_{3} v_{0} v_{1}>,<v_{2} v_{0} v_{4} v_{2}>$ respectively and $v_{0}$ is the common vertex.

case:(ii) One of $v_{1}, v_{2}$ is $v_{0}$.

Without loss of generality assume that $v_{1}=v_{0}$. Since $\left\{v_{0}\right\}$ is a dominating set for $G, v_{0} v_{2} \in E(G)$. Then $\left\langle v_{0} v_{2} v_{3} v_{0}>\right.$ is a triangle in $G$ for some $v_{3}$ in $V-\left\{v_{0}, v_{2}\right\}$. Thus $v_{0}, v_{2}$ lie on the same triangle in $G$.

Therefore from case(1) and case(2), any two vertices in $G$ lie on the same triangle or they lie on different triangles having a common vertex.

Hence by the characterization theorem for semi complete graphs, $G$ is semi complete.

Assume that the converse holds. 
Since $G$ is semi complete, by Theorem(3.2) $G^{N}$ is complete.

$\Rightarrow \gamma\left(G^{N}\right)=1$

Hence $\gamma_{g n}(G)=1$. $G$ be a semi complete graph. Then $\gamma_{g n}(G)=1$ iff $G$ is a union of triangles having a common vertex.

Proof: Assume that $\gamma_{g n}(G)=1$

$\left.\Rightarrow \gamma_{(} G\right)=1, \gamma_{g n}(G)=1$. Let $D=\left\{v_{0}\right\}$ is a dominating set for $G, G^{N}$ for some $\left\{v_{0}\right\}$ in $V$.

Let $\left\{v_{1}, v_{2} \in E(G)\right\}$.

Case:1. Suppose $v_{1} \neq v_{0} \neq v_{2}$.

Then $<v_{1} v_{0} v_{2} v_{1}>$ is a triangle in $G$.

Case:2. One of $v_{1}, v_{2}$ is $v_{0}$.

Without loss of generality assume that $v_{1}=v_{0}$. since $v_{0} v_{2}$ is an edge in $G$ and $G$ is semi complete there is a $v_{3} \in G$ such that $\left\langle v_{0} v_{2} v_{3} v_{0}>\right.$ is a triangle in $G$.

Since $v_{1} v_{2}$ is an arbitrary edge, each edge lies on a triangle having $v_{0}$ as the common vertex.

Assume that the converse holds.

By our assumption $\gamma(G)=1$. Then by Theorem $(3.3) \gamma_{g n}(G)=1$.

Theorem 3.3. $G$ be a connected graph and $H$ be a spanning subgraph of $G$, then $\gamma_{g n}(G) \leq \gamma_{g n}(H)$.

Theorem 3.4. $G$ be a connected graph, then $\gamma_{g n}(G)=n$ iff $G=K_{2}$.

Proof: Assume that $\gamma_{g n}(G)=n$.

Suppose that $\operatorname{diam}(G) \geq 2$. Let $\left\langle v_{1} v_{2} \ldots v_{k-1} v_{k}>(k \geq 3)\right.$ be the diammetral path in $G$. Then $V-\left\{v_{1}\right\}$ or $V-\left\{v_{k}\right\}$ is a gnd - set in $G$, which is a contradiction to our assumption. So,diam $(G)=1$. This implies $G=K_{n}(n \geq 2)$. 
By Corollary.3.3. $n=2$ (i.e) $G=K_{2}$. The converse part is clear.

Theorem 3.5. $G$ be a connected graph, then $\gamma_{g n}(G)=n-1$ iff $G=P_{3}$.

Proof: Assume that $\gamma_{g n}(G)=n-1$.

Suppose that $\operatorname{diam}(G) \geq 3$. Let $\left\langle v_{1} v_{2} \ldots v_{k-1} v_{k}>(k \geq 4)\right.$ be the diammetral path in $G$. Then $V-\left\{v_{1}, v_{k}\right\}$ is a gnd - set in $G$, which is a contradiction to our assumption. Hence $\operatorname{diam}(G) \leq 2$. By the above Theorem and by our assumption $\operatorname{diam}(G) \neq 1$. So $\operatorname{diam}(G)=2$.

If $G=P_{3}$, then $\gamma_{g n}(G)=n-1$.

Suppose $G \neq P_{3}$. Form a spanning tree $G^{\prime}$ of $G$. Clearly $\operatorname{diam}\left(G^{\prime}\right) \geq 3$. This implies $\gamma_{g n}(G)<n-1$, a contradiction. Hence our supposition is false.

The converse part is clear.

Theorem 3.6. $G$ be a connected graph of order $n \geq 4$, then $\gamma_{g n}(G)=n-2$ iff $G=C_{n}(3 \leq n \leq 5)$ or $G=P_{n}(n=4,5)$ or $G=S_{3}$ or $G$ is isomorphic to

Proof: Assume that $\gamma_{g n}(G)=n-2$.

Suppose $\operatorname{diam}(G) \geq 5$. Form a spanning tree $G^{\prime}$ of $G$. Clearly $\operatorname{diam}\left(G^{\prime}\right) \geq$ 5 .

If $G^{\prime}$ has more than two pendant vertices, then by Theorem.3.5. $V\left(G^{\prime}\right)-$ $\left\{\right.$ pendant vertices in $\left.G^{\prime}\right\}$ is a gnd - set of $G$ of cardinality less than $n-2$. Hence a contradiction to our assumption. 
If $G^{\prime}$ has exactly two pendant vertices, then $G^{\prime}=\left\langle v_{1} v_{2} \ldots v_{k-1}, v_{k}\right\rangle$ $(k \geq 6)$. By Theorem 3.5. $V-\left\{v_{1}, v_{4}, v_{k-3}, v_{k}\right\}$ is a gnd - set of $G$ of cardinality less than $n-2$, a contradiction.

Hence $\operatorname{diam}(G) \leq 4$.

Suppose $\operatorname{diam}(G)=4$. Let $P_{5}=<v_{1} v_{2} v_{3} v_{4} v_{5}>$ be a diammetral path in $G$.

Case:1. $V(G)=V\left(P_{5}\right)$. $n-2$.

We have two possibilities $G=P_{5}$ or $G \neq P_{5}$. If $G=P_{5}$, then $\gamma_{g n}(G)=$

Suppose $G \neq P_{5}$.

If $g(G)=3$, then $\gamma_{g n}(G)=2<n-2$ which is a contradiction.

If $g(G)=4$, then $G$ is isomorphic to

In either case $\gamma_{g n}(G)=2<n-2$.

If $g(G)=5$, then $G \cong C_{5}$. This implies $\gamma_{g n}(G)=n-2$.

Case:2. $V(G) \neq V\left(P_{5}\right)$.

Form a spanning tree $G^{\prime}$ of $G$. Clearly $\operatorname{diam}\left(G^{\prime}\right) \geq 4$ and $G^{\prime}$ has atleast three pendant vertices, which implies $\gamma_{g n}(G)<n-2$, a contradiction.

Suppose $\operatorname{diam}(G)=3$.

Let $P_{4}=<v_{1} v_{2} v_{3} v_{4}>$ be a diammetral path in $G$. 
Case:1. $V(G)=V\left(P_{4}\right)$. $n-2$.

We have two possibilities $G=P_{4}$ or $G \neq P_{4}$. If $G=P_{4}$, then $\gamma_{g n}(G)=$

Suppose $G \neq P_{4}$. Clearly $g(G) \leq 4$.

If $g(G)=3$, then $G$ is a union of triangles having a common vertex or $G$ is isomorphic to $H$. In the former case, by Corollary.3.4. we get $\gamma_{g n}(G)=1<n-2$ a contradiction. In the later case we have $\gamma_{g n}(G)=n-2$.

If $g(G)=4$, then $\gamma_{g n}(G)=n-2$ and $G=C_{4}$.

Case:2. $V(G) \neq V\left(P_{4}\right)$.

Then as in the case:2 of $\operatorname{diam}(G)=4$ we get a contradiction.

Suppose $\operatorname{diam}(G)=2$.

Let $P_{3}=<v_{1} v_{2} v_{3}>$ be a diammetral path in $G$.

Case:1. $V(G)=V\left(P_{3}\right)$.

We have two possibilities $G=P_{3}$ or $G \neq P_{3}$. If $G=P_{3}$, then $\gamma_{g n}(G)>$ $n-2$ a contradiction. If $G \neq P_{3}$, then $G=C_{3}$. Thus $\gamma_{g n}(G)=n-2$.

Case:2. $V(G) \neq V\left(P_{3}\right)$.

Form a spanning tree $G^{\prime}$ of $G$. Clearly $\operatorname{diam}\left(G^{\prime}\right) \geq 2$.

If $G^{\prime}$ has more than one internal vertex then, $V(G)-\left\{\right.$ pendant vertices of $\left.G^{\prime}\right\}$ is a gnd - set of $G^{\prime}$. Hence a gnd - set of $G$ of cardinality less than $n-2$ a contradiction.

If $G^{\prime}$ has exactly one internal vertex ((i.e) $\left.v_{2}\right)$, then $G^{\prime} \cong S_{n}(n \geq 2)$. If $n \geq 4$, then $\gamma_{g n}(G)<n-2$ a contradiction. If $G^{\prime}=S_{2}$ again we get a contradiction. So $G^{\prime}=S_{3}$. This implies $G=S_{3}$ or $G$ is a union of triangles having a common vertex or $G$ is isomorphic to $H$. If $G$ is a union of triangles having a common vertex then by Corollary.3.4. we get a contradiction to $\gamma_{g n}(G)=n-2$. In the remaining cases the equality holds good. 
Suppose $\operatorname{diam}(G)=2$. Hence $G=K_{n}(n \geq 2)$. If $n=3$ we have $\gamma_{g n}(G)=n-2$, otherwise we get a contradiction.

The converse part is clear.

Theorem 3.7. $G$ be a connected graph with $g(G) \neq 3$. Then $\gamma_{g n}(G)=2$ iff there is an edge uv in $G$ with $N(u) \cup N(v)=V$.

Proof: Assume that $\gamma_{g n}(G)=2$. Then there is a $D=\{u, v\}$, a gnd - set of $G$. Clearly uv $\in E(G)$ (if not there is a $w \in V$ which is not dominated by either $u$ or $v$ in $G^{N}$, a contradiction). Also since $D$ is a dominating set for $G N(u) \cup N(v)=V$.

Assume that the converse holds. Let $D=\{u, v\}$. By our assumption $D$ is a dominating set of $G$. Since $g(G) \neq 3, D$ is a dominating set of $G^{N}$. So $D$ is a gnd - set for $G$. Since $g(G) \neq 3, G$ cannot be semi complete. Then by the Corollary.3.3., $D$ is a gnd - set of minimum cardinality. Hence $\gamma_{g n}(G)=2$.

Theorem 3.8. $G$ be a semi complete graph and $D \subset V$. Then $D$ is a dominating set for $G$ iff $D$ is a gnd - set for $G$.

Proof: Suppose that $D$ is a dominating set for $G$. Let $v_{1} \in V-D$. Then there is a $v_{2} \in D$ such that $v_{1} v_{2}$ is an edge in $G$. Since $G$ is semi complete there is $v_{3} \in D-\left\{v_{1}, v_{2}\right\}$ such that $v_{1} v_{2} v_{3} v_{2}$ is a triangle in $G$. Thus $v_{1} v_{3}, v_{3} v_{2}$ are edges in $G$

$\Rightarrow v_{1} v_{2} \in E(G), v_{2} \in D$

Hence $D$ is a gnd - set for $G$. The converse part is clear.

Theorem 3.9. $G$ be a connected graph and $D \subset V$ is independent. Then $D$ is a gnd - set for $G$ iff $D$ is a restrained dominating set for $G$.

Proof: Assume that $D$ is a restrained dominating set for $G$. 
Let $v_{1} \in V-D$. By our assumption there is $v_{2}$ in $V-D, v_{3}$ in $D$ such that $v_{1} v_{2}, v_{1} v_{3}$ are in $E(G)$. Since $v_{2} \in V-D$ and $D$ is a dominating set for $G$ there is a $v_{4}$ in $D$ such that $v_{2} v_{4} \in E(G)$.

If $v_{1} v_{4}$ is in $G$, then $<v_{1} v_{2} v_{4} v_{1}>$ is a path in $G \Rightarrow v_{1} v_{4} \in E\left(G^{N}\right)$.

If $v_{1} v_{4}$ is not in $G$, since $v_{2}$ is a common neighbour to $v_{1}, v_{4} \Rightarrow v_{1} v_{4} \in$ $E\left(G^{N}\right)$.

$D$ is a gnd - set of $G$.

Conversely assume that $D$ is a gnd - set for $G$.

Let $v_{1} \in V-D$, by our assumption there is a $v_{2}$ in $D$ such that $v_{1} v_{2}$ is in $G$. Since $D$ is a dominating set for $G^{N}$ there is a $v_{3}$ in $V-D$ such that $v_{1} v_{3}$ is an edge in $G$. Otherwise we get a contradiction to that $D$ is independent.

Since $v_{1} \in V-D$ is arbitrary, $D$ is a restrained dominating set for $G$.

If $G \neq K_{m, n}$ and $G$ is bipartite, then none of the partite sets of $G$ can form a gnd - set for $G$.

Proof: Let $V_{1}, V_{2}$ be the partite sets for $G$.

Assume that $V_{1}$ is a gnd - set for $G$.

Let $v_{1} \in V-V_{1}\left(\Rightarrow v_{1} \in V_{2}\right)$. Since $V_{1}$ is a gnd - set for $G$, by the characterization theorem for gnd - set there is a $v_{2}$ in $G$ and $v_{3}, v_{4}$ in $V_{1}$ such that $<v_{3} v_{1} v_{2} v_{4}>$ is a path in $G$.

case:(i) Suppose $v_{2}=v_{3}$.

Then $\left\langle v_{1} v_{3} v_{4}>\right.$ is a path in $G$, where $v_{3}, v_{4} \in V_{1}$ which is a contradiction to that $G$ is bipartite.

case:(ii) Suppose $v_{2} \neq v_{3}$.

Then $v_{2} \in V_{1}$ (or) $V_{2}$ we get a contradiction to that $G$ is bipartite.

Hence $V_{1}$ is not a gnd - set for $G$.

Similarly $V_{2}$ is not a dominating set for $G$. 
$G=C_{n} O K_{2}$. If $D \subset V$ is a minimum gnd - set of $G$, then $D$ is independent iff $D=\left\{v \in V(G): d_{G}(v)=1\right\}$.

Proof: Assume that $D$ is independent.

Suppose that $v_{1} \in D$ and $d_{G}(v) \neq 1$. Since $D$ is a minimum independent gnd - set for $G$ there is a $v_{2} \in V-D$ such that $v_{1} v_{2}$ is an edge in $G$ and $d_{G}\left(v_{2}\right)=1$.

Also there is an edge in $G$ such that $v_{1} v_{3}$ is an edge in $G$. Hence a contradiction. Thus $v_{1} \in D$, then $d_{G}\left(v_{1}\right)=1$.

Hence $D=\left\{v \in V(G): d_{G}(v)=1\right\}$.

The converse part is clear.

\section{References}

[1] Bondy J. A. and Murthy, U. S. R., Graph theory with Applications, The Macmillan Press Ltd, (1976).

[2] R. C. Brigham, R. D. Dutton, On Neighbourhood Graphs, J. Combin. inform. System Sci, 12, pp. 75-85, (1987).

[3] G. S. Domke, etal., Restrained Domination in Graphs, Discrete Mathematics, 203, pp. 61-69, (1999).

[4] T. W. Haynes, S. T. Hedetneimi, P. J. Slater, Fundamentals of Dominations in Graphs Marcel Dekker, New York, (1988).

[5] I. H. Naga Raja Rao, S. V. Siva Rama Raju, On Semi-Complete Graphs, International Journal Of Computational Cognition, Vol.7(3), pp. 50-54, (2009).

[6] D. F. Rall, Congr. Numer., 80, pp. 89-95, (1991).

[7] E. Sampathkumar, H. B. Walikar, The connected Domination Number of a Graph, J. Math. Phy. Sci, Vol.13, pp. 607-613, (1979).

[8] E. Sampathkumar,The global domination number of a graph, $J$. Math.Phy. Sci, Vol. 23 (5), (1989). 
S. V. Siva Rama Raju

Department of Mathematics

Ibra College of Technology

Ibra,

Sultanate of Oman

e-mail:shivram2006@yahoo.co.in

and

I. H. Nagaraja Rao

Department of Mathematics

G. V. P. P. G. Courses

Visakhapatnam,

India

e-mail : ihnrao@yahoo.com 